\title{
Potencialidades e fragilidades da Educação e do Trabalho Interprofissional em saúde: perspectivas de profissionais do Nordeste brasileiro
}

\author{
Potentialities and weaknesses of \\ Education and Interprofessional Work in \\ health: perspectives of professionals in \\ Northeast Brazil
}

\section{Emanuella Pinheiro de Farias Bispo' ${ }^{1}$ (]) Rosana Aparecida Salvador Rossit ${ }^{2}$ (C)}

${ }^{1}$ Autora para correspondência. Universidade Estadual de Ciências da Saúde de Alagoas (Maceió). Alagoas, Brasil. emanuellapinheirofbispo@gmail.com universidade Federal de São Paulo (São Paulo), São Paulo, Brasil. rorossit@hotmail.com

RESUMO | INTRODUÇÃO: A Organização Mundial da Saúde reconhece que a EIP é uma estratégia inovadora que proporciona a prática colaborativa. A mesma ocorre quando duas ou mais profissões aprendem sobre os outros, com os outros e entre si. OBJETIVO: Apresentar a análise das potencialidades e fragilidades da educação e do trabalho interprofissional na perspectiva de profissionais da saúde do setor público do Nordeste brasileiro. MÉTODOS E MATERIAIS: Trata-se de uma pesquisa de campo exploratória, com abordagem qualitativa. Os dados foram coletados com 189 participantes da área da saúde de diversas formações na área de saúde do Nordeste brasileiro. Os dados coletados foram inseridos em quadros sinópticos e organizados em categorias temáticas. RESULTADOS: Os conteúdos expressos no espaço aberto do formulário eletrônico foram analisados a partir de dois eixos temáticos que emergiram dos resultados da pesquisa e nortearam as análises: Importância, desafios e possibilidades da Educação e Trabalho Interprofissional; e Formação em Saúde para a prática Interprofissional. Os conteúdos expressos apontam para a necessidade de mudanças curriculares nas graduações dos cursos da saúde, no intuito de favorecer uma formação voltada para a interprofissionalidade e com capacidade para atuar em equipes colaborativas. CONCLUSÃO: Foram identificados déficits na formação acadêmica e educação permanente para o fortalecimento da EIP para o fortalecimento das práticas interprofissionais e, consequentemente, para o fortalecimento das práticas interprofissionais.

PALAVRAS-CHAVE: Educação interprofissional. Aprendizado colaborativo. Formação. Equipe de assistência ao paciente.

\begin{abstract}
INTRODUCTION: The World Health Organization recognizes that IPE is an innovative strategy that provides collaborative practice. The same occurs when two or more professions learn about others, with others and with each other. OBJECTIVE: To present the analysis of the strengths and weaknesses of education and interprofessional work from the perspective of health professionals in the public sector in Northeast Brazil. METHODS: It is exploratory field research with a qualitative approach. Data were collected from 189 health care participants from different backgrounds in health care in Northeast Brazil. The collected data were inserted in synoptic tables and organized into thematic categories. RESULTS: The contents expressed in the open space of the electronic form were analyzed from two thematic axes that emerged from the research results and guided the analysis: Importance, challenges, and possibilities of Education and Interprofessional Work; and Health Training for Interprofessional practice. The contents expressed point to the need for curricular changes in the graduations of health courses in order to favor training aimed at interprofessional and with the capacity to work in collaborative teams. CONCLUSION: Deficits in academic training and continuing education have been identified to strengthen IPE to strengthen interprofessional practices and, consequently, to strengthen interprofessional practices.
\end{abstract}

KEYWORDS: Interprofessional education. Collaborative learning. Training. Patient care team.

Como citar este artigo: Bispo EPF, Rossit RAS. Potencialidades e

fragilidades da Educação e do Trabalho Interprofissional em saúde: perspectivas de profissionais do Nordeste brasileiro. Rev Inter Educ Saúde. 2021;5(1):79-91. http://dx.doi.org/10.17267/2594-7907ijhe. v5i1.3717 


\section{Introdução}

A educação interprofissional em saúde (EIP) vem ganhando grande visibilidade e valorização ao redor do mundo por estar orientada por marcos teórico-conceituais e metodológicos coerentes com o desafio de formar profissionais de saúde mais preparados à colaboração e ao efetivo trabalho em equipe. ${ }^{1}$

A Organização Mundial da Saúde ${ }^{2}$ reconhece que a EIP é uma estratégia inovadora que proporciona a prática colaborativa e desempenhará um papel essencial na redução da crise mundial na força de trabalho em saúde. A EIP é um passo importante de preparo dos profissionais para responder às necessidades de saúde loco regionais.

Os princípios da EIP se aplicam tanto para a formação profissional quanto para a educação permanente em saúde ${ }^{3}$ e visam a formação do trabalho colaborativo, que é fundamental para a integralidade do cuidado. ${ }^{4}$

A EIP propõe mudanças nos níveis educacional, profissional e organizacional, e oportuniza uma formação que potencializa o desenvolvimento de competências colaborativas. $\frac{15}{5}$

De acordo com Reeves, Xyrichis, Zwarenstein ${ }^{6}$, a colaboração compreende o compartilhamento, parceria, interdependência, poder e envolve a reflexão e ação mútua em busca de respostas às necessidades de saúde dos usuários.

Para Reeves ${ }^{1}$, as equipes interprofissionais são constituídas por duas ou mais categorias profissionais que compartilham conhecimentos e práticas para o planejamento, execução de projetos e atividades no seu contexto de trabalho.

Peduzzi ${ }^{7}$ e demais pesquisadores indicam que o trabalho em equipe interprofissional é entendido como uma forma de trabalho coletivo que compreende uma relação recíproca entre as intervenções técnicas e as interações dos múltiplos atores envolvidos, e necessita, de um lado, a articulação das ações das diferentes áreas profissionais e de outro, a complementaridade entre agir instrumental e agir comunicativo.

O resultado da aprendizagem na perspectiva da EIP e do trabalho colaborativo é ter um profissional de saúde colaborativo e preparado para a prática interprofissional, que aprendeu a trabalhar em equipe e tem competências para este fim. $\frac{8}{}$ Entretanto, para que haja informações sobre o nível de colaboração dentro dos ambientes de atenção à saúde, torna-se necessário ter mecanismos e instrumentos apropriados para avaliar esta prática.

De acordo com Peduzzị, para a construção de um ambiente favorável para o aprendizado interprofissional é necessário desenvolver estudos que permitam conhecer que tipo de educação funciona melhor, em que circunstâncias e como esta formação pode impactar nos resultados da atenção à saúde de usuários e população, trabalhadores e organizações.

Assim, o presente estudo teve por objetivo analisar as potencialidades e fragilidades da educação, e do trabalho, interprofissional na perspectiva de profissionais da saúde do Nordeste brasileiro. Este estudo foi realizado no Nordeste por ser parte adaptada da tese de doutoramento "Tradução e a adaptação transcultural do Assessment of Interprofessional Team Collaboration Scale II - AITCS II para profissionais da área de saúde destinada à realidade brasileira" desenvolvida no Programa de Pós-Graduação Interdisciplinar em Ciências da Saúde, UNIFESP-BS e apresenta o estudo referente à tradução, adaptação transcultural e validação do Assessment of Interprofessional Team Collaborationscale II (AITCS II, ORCHARD, 2015) para o português brasileiro.

Espera-se que os aspectos apresentados nessa produção possam desencadear reflexões e ações para a qualificação da formação e do trabalho em saúde na perspectiva da interprofissionalidade.

\section{Percurso metodológico}

O campo determinado para este estudo compreendeu os serviços de saúde do setor público nos quais profissionais de diferentes áreas de formação atuam nos seguintes setores: 1) Atenção Básica, incluindo a Estratégia de Saúde da Família (ESF) e as equipes dos Núcleos de Apoio à Saúde da Família e Atenção Básica (NASF-AB); 2) Equipes dos Centros de Atenção Psicossocial (CAPS); e, 3) Equipes de Saúde do Trabalhador (EST). Além de contar com a participação de profissionais de saúde que exercem, concomitantemente, a atuação em cenários do trabalho 
em saúde e a docência em cursos de graduação na área da saúde (Serviço Social, Cirurgião-dentista, Enfermagem, Farmácia, Fonoaudiologia, Fisioterapia, Medicina, Nutrição, Psicologia, Terapia ocupacional, Agente comunitário de saúde, Auxiliar em saúde bucal, Técnico de Enfermagem), pois acredita-se que o trabalho docente influencia diretamente no processo de trabalho nos serviços de saúde.

Os campos da ESF, NASF-AB, CAPS e Saúde do Trabalhador são locais com potencial para o desenvolvimento do trabalho em equipe e da prática colaborativa. Estes campos favorecem a construção de projetos coletivos, principalmente com a utilização de instrumentos como a Clínica Ampliada, Matriciamento e o Projeto Terapêutico Singular (PTS), baseando-se nos princípios e diretrizes do Sistema Único de Saúde (SUS) e no conceito ampliado de saúde. Estes recursos utilizam um conjunto de propostas e condutas terapêuticas articuladas para um sujeito, individual ou coletivo, resultado da discussão coletiva de uma equipe de profissionais composta por diferentes formações em saúde. $\frac{10}{}$

Nestes serviços se reconhece a integralidade da atenção e a necessidade de um cuidado ancorado na concepção ampliada de saúde, sendo, portanto, escolhido como um potencial espaço para analisar o nível de colaboração interprofissional entre os membros de uma equipe.

Para a composição da amostra, inicialmente, foi realizada uma pesquisa nos sites das Secretarias Estaduais de Saúde dos nove estados da região Nordeste do Brasil com o intuito de ter acesso ao endereço eletrônico destas Secretarias. Também foi realizada uma pesquisa nos sites oficiais das Secretarias Municipais de Saúde das capitais dos nove respectivos estados.

Além do contato pelo endereço eletrônico, a pesquisadora manteve contato por telefone com algumas Secretarias de Saúde, com o intuito de reforçar a importância dessa colaboração na pesquisa. Também utilizou as redes sociais, por meio de aplicativo/ software para smartphones, utilizados para troca de mensagens de mensagem instantaneamente, para intensificar o convite. Os contatos de alguns coordenadores de equipes de saúde foram disponibilizados pelas Secretarias de saúde, o que favoreceu maior aproximação e a colaboração com a pesquisa, repassando aos profissionais das Unidades de saúde, o convite com o link de acesso ao formulário pelo aplicativo.

Trata-se de uma pesquisa de campo exploratória, com abordagem qualitativa. ${ }^{11}$

Minayo ${ }^{11}$ enfatiza que a amostra de uma pesquisa deve estar vinculada à dimensão do objeto que, por sua vez, articula-se com a escolha do grupo ou dos grupos a serem abordados. A autora afirma que o tamanho da amostra e seu desenho representam um processo ativo de reflexão.

Como critérios de inclusão foram considerados os trabalhadores da saúde do Nordeste brasileiro com atuação em cenários de prática junto a profissionais de diferentes áreas da saúde e que concordaram e assinaram o termo de consentimento livre e esclarecido. Não participaram da pesquisa os trabalhadores da saúde que não concordaram com os termos da pesquisa, ou não estavam disponíveis durante o período de coleta de dados.

Para a composição da amostra, inicialmente, foi realizada uma pesquisa nos sites das Secretarias Estaduais de Saúde dos nove estados da região Nordeste do Brasil com o intuito de ter acesso ao endereço eletrônico destas Secretarias. Também foi realizada uma pesquisa nos sites oficiais das Secretarias Municipais de Saúde das capitais dos nove respectivos estados.

Além do contato pelo endereço eletrônico, a pesquisadora manteve contato por telefone com algumas Secretarias de Saúde, com o intuito de reforçar a importância dessa colaboração na pesquisa. Também utilizou as redes sociais, por meio de aplicativo/software para smartphones, utilizados para troca de mensagens de mensagem instantaneamente, para intensificar o convite. Os contatos de alguns coordenadores de equipes de saúde foram disponibilizados pelas Secretarias de saúde, o que favoreceu maior aproximação e a colaboração com a pesquisa, repassando aos profissionais das Unidades de saúde, o convite com o link de acesso ao formulário pelo aplicativo.

A região Nordeste possui cerca de 60 cursos da área da saúde alocados em instituições públicas (federais e estaduais). Busca direta foi realizada nos sites oficiais destas instituições e contato prévio, tanto por 
telefone quanto por endereço eletrônico foi mantido com os coordenadores dos cursos em busca de docentes que também atuassem nos serviços de saúde pública. As coordenações de curso enviaram os endereços eletrônicos dos seus profissionais de saúde/professores e o convite com o link do formulário online, contendo o TCLE e o ATCS II-BR, foi enviado diretamente para os endereços destes profissionais. Alguns destes profissionais responderam de forma presencial nas dependências da Unidade de Saúde ou da própria instituição de ensino superior.

Comunicação foi estabelecida com Secretarias Estaduais e Municipais de Saúde do nordeste brasileiro, por endereço eletrônico e telefone, para conseguir os contatos de coordenadores das equipes de saúde, para que estes pudessem mediar o envio do convite aos profissionais para o processo de coleta de dados. Os coordenadores repassaram aos trabaIhadores das Unidades de saúde, o convite de participação juntamente com o link de acesso ao formulário eletrônico. As redes sociais, também foram utilizadas para localizar os potenciais participantes e para troca de mensagens instantâneas, para intensificar o convite e a importância da participação.

O instrumento da pesquisa continha a escala AITCS II$\mathrm{BR}^{12}$, que já havia passado pelo processo de validação e adaptação transcultural, e um espaço aberto com enunciado estimulando os participantes a contribuírem com relatos espontâneos sobre potencialidades, fragilidades e sugestões para a formação e o trabalho interprofissional em saúde. Os dados obtidos destes relatos foram analisados e constituem o corpo de conhecimento apresentado neste manuscrito.

A pesquisa foi registrada na Plataforma Brasil e aprovada pelo Comitê de Ética em Pesquisa da Universidade Federal de São Paulo, seguindo os preceitos da Resolução 466/12 de 12 de dezembro de 2012, com o Protocolo de aprovação CAAE: 55262416.5.0000.5505, Parecer $n^{\circ} 1.575 .616$ de 05 de Junho de 2016.

A aplicação do instrumento ocorreu de modo presencial em algumas localidades (estados da Paraíba, Rio Grande do Norte e Alagoas). Nesses locais, o instrumento foi aplicado nas Unidades de Saúde por meio de cópia impressa e respondido após a concordância e assinatura do Termo de Consentimento Livre e Esclarecido. Para os outros estados da região nordeste, a aplicação foi mediante formulário eletrônico. O questionário foi apresentado dessas duas maneiras com o objetivo de atingir um maior número de participantes. Das duas formas de aplicação, o participante auto administrou a resolução do questionário.

Para a organização e análise qualitativa dos dados adotou-se a metodologia de Minayo ${ }^{13}$ e Franco. ${ }^{14} \mathrm{~A}$ análise qualitativa "permite a compreensão de valores culturais e representações de determinado grupo acerca de um tema específico, sob diferentes perspectivas, abordando as relações entre atores sociais e ações implementadas". Para analisar o conteúdo destas mensagens foram utilizadas as Unidades de Registro. ${ }^{15}$

Os dados coletados foram inseridos em quadros sinópticos e organizados em categorias temáticas adotando os seguintes passos: a) leituras das respostas, com o intuito de compreender e apreender o sentido das informações; b) condensação das informações; c) identificação em cada item dos pontos comuns e agrupamento das similaridades, permitindo a construção de categorias temáticas de análise; e, d) análise reflexiva das respostas significativas para o estudo, com o intuito de aprofundamento do objeto..$^{13}$

Para a organização dos conteúdos expressos, os relatos foram compilados em um único documento. Inicialmente, foi realizada uma pré-análise por meio da leitura exaustiva, em que foi realizado o contato profundo com o material coletado. O material coletado foi lido e relido por observador convidado para contraprova. Essas leituras permitiram a impregnação dos sentidos e a compreensão das particularidades contidas nos relatos, como também incorporar os significados ampliados dos contextos apresentados. Após esse primeiro contato com os relatos e o pareamento com os objetivos do estudo foram extraídas as Unidades de Contexto (UC), que representam a parte mais importante e significativa dos conteúdos. ${ }^{13}$ De cada UC emergiu uma ou mais Unidades de Registro (UR) as quais, a partir de seus significados, foram organizadas e agrupadas em categorias. Os dados foram organizados em um quadro sinóptico contendo as UC, UR, categorias e subcategorias, e por fim, interpretados e discutidos à luz do referencial teórico. A discussão das categorias e subcategorias foi realizada pelas autoras do estudo. 
Figura 1. Esquema do processo de análise qualitativa das respostas abertas fornecidas pelos participantes

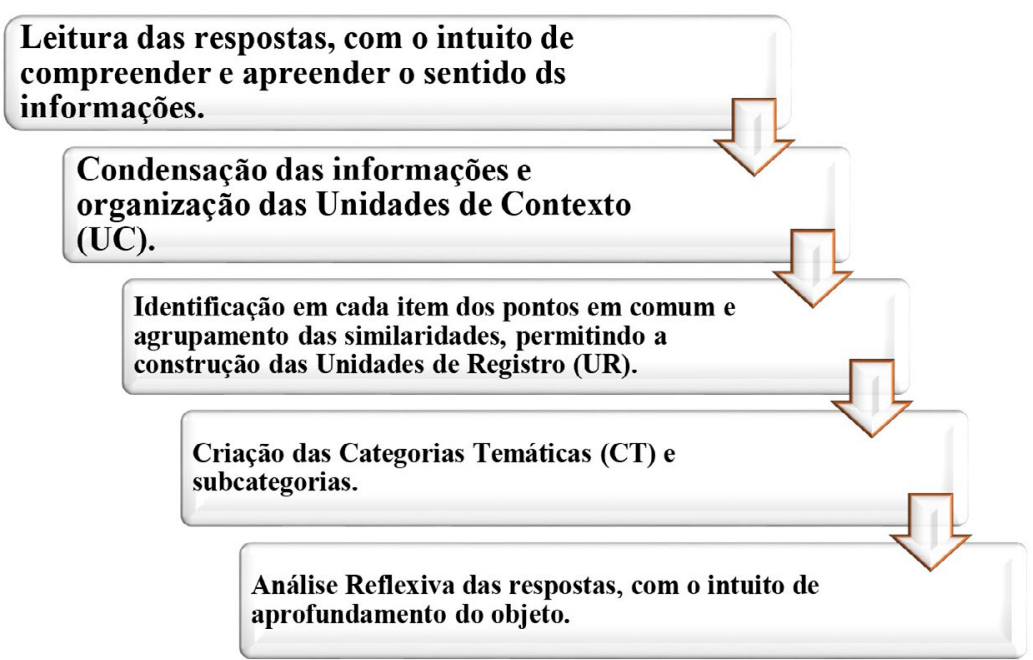

Fonte: Elaborado pelo Autor

\section{Resultados e discussão}

Cerca de 1.000 contatos foram estabelecidos com o envio do convite para participação na pesquisa. Este número de contatos foi a média de profissionais que foram disponibilizados pelos serviços. O total de participantes foi de 189 (18,9\%) profissionais de saúde que responderam à questão aberta. Todos os questionários foram respondidos e utilizados para estudo nesta pesquisa.

Os dados foram coletados com 189 participantes de diversas formações na área de saúde: Serviço Social, Odontologia, Enfermagem, Farmácia, Fonoaudiologia, Fisioterapia, Medicina, Nutrição, Psicologia, Terapia ocupacional, Agente comunitário de saúde, Auxiliar em saúde bucal, Técnico de Enfermagem do Nordeste brasileiro que atuam nos cenários da AB, ESF, CAPS, EST, NASF-AB e em cursos de graduação na área da Saúde.

Os conteúdos expressos no espaço aberto do instrumento de pesquisa foram analisados qualitativamente utilizando-se a técnica da análise de conteúdo na modalidade temática. Para preservar a identificação dos participantes, os trechos foram denominados por expressões alfanumérica (P1, P2, P3 e assim por diante).

Considerando que a EIP e as práticas colaborativas são temas que vêm sendo amplamente discutidos no cenário mundial, com ênfase na reformulação do modelo de formação profissional em saúde 2 , discute-se a seguir os conteúdos expressos pelos profissionais no espaço aberto do AITCS II-BR ${ }^{12}$, e a identificação das competências colaborativas, as potencialidades e fragilidades da formação e do trabalho interprofissional.

Considerando as competências específicas de cada profissão e as comuns a todas as profissões da saúde, identificou-se principalmente, as competências colaborativas para o trabalho interprofissional, que envolve o respeito às outras profissões, o planejamento coletivo, o exercício do diálogo e da tolerância em um ambiente favorável.

Para discutir as competências apresentadas nos conteúdos expressos adotou-se as seguintes competências colaborativas: comunicação interprofissional; cuidado/atenção ao paciente/usuário; esclarecimento de funções/ clarificação de papeis; funcionamento da equipe; liderança colaborativa; resolução de conflitos interprofissionais; valores éticos para a prática interprofissional; responsabilidades e trabalho em equipe. . $16-18^{18}$ 
Dois eixos temáticos (ET) nortearam as análises: ET1 - Importância, desafios e possibilidades da Educação e Trabalho Interprofissional; e, ET2 - Formação em Saúde para a prática Interprofissional. Foram identificadas 189 UC e 211 UR com as respectivas categorias e subcategorias que emergiram.

Na análise temática do ET1 - Importância, desafios e possibilidades da Educação e Trabalho Interprofissional, foram encontradas 124 UC e 137 UR. A partir das UR emergiram quatro categorias: Reconhecimento da importância da interprofissionalidade; Desafios do preparo profissional para a prática interprofissional; Possibilidades da formação profissional para a interprofissionalidade; e, Limitações para o trabalho em equipe.

$\mathrm{Na}$ categoria Reconhecimento das potencialidades da interprofissionalidade (22 UR) emergiram os seguintes conteúdos:

\section{[...] A prática interprofissional colabora de forma} significativa na formação do docente/preceptor, uma vez que cada um pode contribuir com seus conhecimentos e experiências visando tanto o próprio aprendizado e do discente, como os melhores resultados na qualidade de vida dos pacientes. (P26)

[...] Importante e essencial o trabalho interprofissional, pois engrandece a relação entre os profissionais $e$ pacientes com resolutividade satisfatória. (P124)

[...] A prática interprofissional tem um grande potencial para o cuidado em saúde, uma vez que há a contribuição de diferentes visões/conhecimentos que cada membro da equipe possui, que acabam ... se complementando e ampliando as possibilidades de efetivação do cuidado ao usuário. (P128)

De acordo com a OMS2, "existem evidências suficientes para indicar que a prática e a EIP otimizam e fortalecem os serviços de saúde, o que proporciona meIhorias de resultados". Assim, a EIP proporciona uma melhor qualidade na assistência ao favorecer habilidades necessárias para o desenvolvimento de tarefas coletivas $1,19,20$, fortalecendo os serviços de saúde e deixando-os mais resolutivos e integrais no cuidado em saúde.

Os profissionais, ao reconhecerem a importância da interprofissionalidade percebem os benefícios que os profissionais de diferentes formações em saúde, dispostos a transitar entre as áreas específicas de formação, articulam seu saber entre si, na organização do trabalho, compartilhando ações e delegando atividades a outros profissionais, nos moldes de uma prática colaborativa. ${ }^{21}$

Outra vantagem identificada refere-se ao respeito mútuo, o vínculo e o tratamento humanizado entre os profissionais. Costa ${ }^{22}$ fala sobre a importância do estabelecimento dos vínculos profissionais e subsídios para a ampliação da saúde, como essenciais para a prática interprofissional. Para tanto, são necessárias mudanças referentes à comunicação, à socialização dos papéis profissionais e ao processo de trabalho em saúde.,2,22,23

Assim, observa-se que a interprofissionalidade, quando executada de maneira efetiva, proporciona avanços não apenas na perspectiva do cuidado aos usuários, mas também, proporciona vantagens no que diz respeito às relações interpessoais entre os profissionais dos serviços, o respeito e a comunicação para que o efetivo trabalho em equipe acontecer nos diferentes cenários de atenção à saúde. Existe o fortalecimento do vínculo, da ajuda mútua, do apoio recíproco e uma relação humanizada para devolver o trabalho coletivo e colaborativo.

Na categoria Desafios do preparo profissional para a prática interprofissional (21 UR) emergiram os seguintes conteúdos:

[...] Muitas são as potencialidades para o ensino interprofissional ... contudo, a dificuldade de entender até onde o saber do outro complementa o seu, dificulta sua utilização na prática. (P4)

[...] A formação na área da saúde deve atentar para a superação de alguns equívocos conceituais na compreensão e aprofundamento dos princípios que determinam a lógica da educação e do trabalho interprofissional em saúde. (P31)

[...] Penso haver dificuldades para a concretização da prática interprofissional nas graduações em saúde nos campi em que os cursos não foram concebidos a partir de uma perspectiva interprofissional. (P81)

\section{[...] Percebo certa fragilidade e resistência por parte de} alguns profissionais em implementar ao seu cotidiano profissional uma prática colaborativa, visando compartilhar conhecimentos entre a equipe. (P241) 
De acordo com os conteúdos expressos observa-se a necessidade de compreensão do conceito do trabalho interprofissional em saúde e da consistência teórico-conceitual e metodológica. Desse modo, os resultados da aprendizagem na EIP devem atender às necessidades de todas as profissões envolvidas, tendo como resultado um profissional de saúde colaborativo preparado para a prática e que aprendeu como trabalhar em uma equipe interprofissional tendo competência para este fim. 1,24

Para compreender a coordenação das práticas interprofissionais é essencial o entendimento do conceito do trabalho interprofissional em saúde. Os participantes indicam a necessidade emergente de aprendizagens e vivências da interprofissionalidade durante seus processos formativos, nas diferentes graduações em saúde e na educação permanente. O modelo biomédico, fragmentado, segmentado, individualista caracteriza a percepção sobre os modelos de formação vigentes, como responsável pelo modelo de prática profissional das equipes de trabalho. Assim, a prática profissional existente reflete a formação recebida e vivenciada.

Para a OMS2 a EIP estabelece o cenário em que a formação deve ocorrer - "é quando estudantes ou profissionais de dois ou mais profissões, ou núcleos profissionais, aprendem sobre os outros, com os outros e entre si".

Outro desafio refere-se ao lugar do docente e da formação em saúde. O professor pode ser o mediador nas situações de aprendizagem que necessitem da EIP. Para tanto, é preciso um conjunto de dimensões que vão desde as experiências prévias, a intencionalidade para o trabalho interprofissional, a flexibilidade e a criatividade para vivenciar as situações de maneira compartilhada. $\underline{25}$

Para o reconhecimento e compreensão da importância e da implementação do trabalho interprofissional na superação dos desafios da saúde para uma prática colaborativa eficaz, a experiência no desenvolvimento de programas de EIP para docentes e equipes, ressalta a importância de um planejamento integrado e coletivo, sendo necessária uma representação efetiva de diferentes profissões.
Outra questão desafiante nas práticas em saúde refere-se à responsabilidade dos gestores. A gestão passa a ser responsável pela transição de cuidados com o intuito de formar uma equipe preparada para a prática colaborativa que responda às necessidades de saúde dos indivíduos e dos cuidados naquele determinado território. 19

Assim, observa-se que entre os principais desafios do preparo do profissional para a interprofissionalidade estão a formação em saúde, os conhecimento teórico-conceitual e metodológico sobre a EIP e a colaboração interprofissional na perspectiva de instrumentalizá-los para implementar transformações no trabalho em saúde, além de aspectos atitudinais, que envolvem a relutância e resistência de alguns profissionais para produzir mudanças de percepção e de comportamento no cotidiano do trabalho, para a constituição de equipes resolutivas diante das complexas demandas de saúde.

Na categoria Possibilidades de formação para a interprofissionalidade (29 UR) emergiram os seguintes conteúdos:

[...] Criar disciplinas integradas durante toda a graduação e (realizar) visitas clinicas com estudantes de diferentes cursos. (P7)

[...] Reuniões e treinamentos devem ser considerados com frequência e conteúdo direcionado a essas fragilidades, que devem ser reconhecidas pelo líder, para que a equipe trabalhe em unidade e de forma homogênea. (P24)

[...] Investir em mudanças nas políticas de reorientação da formação dos profissionais de saúde, com maior estímulo à interprofissionalidade, fomento às mudanças curriculares e adoção da EIP nas dinâmicas dos cursos da saúde e na valorização de iniciativas que melhorem as relações interpessoais e interprofissionais na formação e no trabalho em saúde. (P31)

Os participantes apontam questões referentes às possibilidades de implementação de ações direcionadas à formação para a interprofissionalidade, como: formação em saúde voltada para a EIP; reuniões e treinamentos das equipes de saúde que trabalham de forma coletiva e integrada; comunicação efetiva entre os profissionais; e, a prática colaborativa em saúde. 
Os participantes relatam, também, sobre a necessidade de uma formação interprofissional, ainda na graduação, com matrizes curriculares potentes para a Educação Interprofissional.

Observa-se a importância da cooperação e da ajuda mútua como essenciais para possibilitar a interprofissionalidade. Neste contexto, é importante destacar que o enfoque da EIP é modificar atitudes e percepções na equipe, melhorar a comunicação entre profissionais, reforçar a competência colaborativa e construir relações dialógicas. ${ }^{26}$ Estes pontos são fortalecidos através de uma formação em saúde voltada para EIP com vistas à integralidade do cuidado e por meio de reuniões e planejamentos coletivos que favorecem o diálogo e a construção coletiva.

De acordo com Peduzzi ${ }^{21}$, profissionais de diferentes formações em saúde articulam seu saber entre si, na organização do trabalho, compartilhando ações e delegando atividades a outros profissionais, nos moldes de uma prática colaborativa.

A interprofissionalidade está vinculada à noção do trabalho em equipe de saúde, marcado pela reflexão sobre os papéis profissionais e a negociação nos processos decisórios, com respeito às singularidades e diferenças dos diversos núcleos de saberes e práticas profissionais. Extrapolar o trabalho em equipe multiprofissional para a perspectiva interprofissional meIhora a produção do cuidado aos usuários. 27

Deste modo, é possível considerar as seguintes possibilidades com potencial para a interprofissionalidade: a) práticas colaborativas e produtivas em saúde; b) melhoria dos resultados obtidos, considerando a integralidade do cuidado e o usuário como centro da atenção; c) comunicação dialógica e aumento da confiança no ambiente de trabalho; d) melhoria do acesso aos de saúde de maneira mais humanizada; e, e) incluir os usuários, familiares e comunidade na tomada de decisões sobre os projetos terapêuticos, para otimizar a adesão aos cuidados de saúde.

Outros aspectos emergem nos relatos: currículo integrado nas graduações em saúde; qualificação nos serviços, incluindo profissionais e gestão em saúde. Estas iniciativas proporcionam avanços importantes no que concerne a interprofissionalidade, pois são propostas de envolvem a formação em saúde desde a graduação até a educação permanente que estão ativos e desempenham, frequentemente, ações fragmentadas, com pouco integração com os demais profissionais e distantes dos princípios e pressupostos da EIP e da colaboração interprofissional.

Reflete-se, assim, que com os avanços há necessidade de incorporar novos modelos de currículo na formação em saúde, com módulos ou disciplinas que proporcionem experiências interprofisisonais. Uma vez que a saúde é ampliada, necessita-se também ampliar as possibilidades formativas, com abordagens e planejamentos que viabilizem o aprender junto para poder atuar junto nos cenários do trabalho em saúde. A EIP configura-se como uma estratégia potente para expandir os saberes e fazeres profissionais, com destaque aos aspectos atitudinais.

A implantação da EIP depende de um conjunto de iniciativas que precisam estar articuladas, mantendo forte relação de interdependência entre três dimensões: macro, meso e micro. 28,29

No nível macro encontram-se as políticas de saúde e educação que reconhecem as bases teórico-conceituais e metodológicas como marcos reorientadores do processo de formação dos profissionais da saúde e do modelo de atenção à saúde. Essas políticas têm importante papel indutor de mudanças nos outros níveis. A dimensão meso se refere a mudanças curriculares, módulos ou disciplinas, que corresponde a EIP em saúde na intencionalidade das propostas pedagógicas dos cursos e instituições. Na dimensão micro, destacam-se as fortes relações interpessoais que fundamentam o desenvolvimento das competências colaborativas. 29,30

Diante desse contexto, é necessário modificar a cultura da formação ainda com forte tendência uniprofissional, centralizada no desenvolvimento das competências específicas das diferentes áreas de conhecimento, sendo as competências colaborativas uma temática nova e uma iniciativa incipiente nos processos de formação em saúde na realidade brasileira.

Na categoria Limitações para o trabalho em equipe (65 UR) emergiram os seguintes conteúdos:

\section{[...] Existe uma relação de poder muito forte nos lideres de minha equipe, não existindo um compartilhar de ideias e liderança. (P11)}




\section{[...] Cada profissional atua de maneira isolada, a partir dos interesses da sua classe. (P13)}

[...] No caso da equipe não creio que ela seja exatamente uma equipe (uma dia foi), mas atualmente, se resume a um conjunto de profissionais interessados em cumprir seus horários e finalizar o atendimento o quanto antes para retornar aos seus consultórios. (P18)

\section{[...] Escassez de conhecimento do papel profissional} do outro e falta de interesse em buscar. Além do comodismo no processo de trabalho. Ou por não conhecer realmente a área que atua ou por desleixo mesmo, no comprometimento de suas funções. (P123)

Nessa categoria temática, as principais limitações para o trabalho em equipe estiveram relacionadas a: dificuldades de comunicação e diálogo aberto; formação individualista e uniprofissional; falta de comprometimento de alguns profissionais; e, fragilidades no sentido de incluir os usuários como corresponsáveis do cuidado em saúde.

Os relatos corroboram com a literatura quanto a importância da comunicação e diálogo para o trabalho em equipe, o que é essencial para a prática interprofissional colaborativa em saúde, sendo importante compreender, neste contexto, o conceito de 'profissional de saúde'. A EIP promove o respeito mútuo, elimina estereótipos e evoca a prática da ética focada no paciente.

O profissional de saúde "é um termo abrangente que inclui indivíduos com conhecimento e/ou habilidades para contribuir com o bem-estar físico, mental e social de uma comunidade... que se refere a todas as pessoas envolvidas em ações cujo objetivo principal é promover a saúde". Incluem-se aqueles que diagnosticam e tratam doenças, profissionais de apoio e gestores da saúde, profissionais com áreas de competência distintas.?

Stone $^{31}$ e Freeth ${ }^{32}$ relatam que a EIP necessita de políticas institucionais de apoio, comunicação coerente entre os participantes, visão compartilhada e compreensão dos benefícios da introdução de um currículo integrado, e, um líder responsável por identificar as limitações e dificuldades.

Os domínios para o aprendizado interprofissional, como: trabalho em equipe; a comunicação dialógica, que é o saber ouvir os membros desta equipe; o aprendizado mútuo; a reflexão sobre esta prática e a integralidade do cuidado, culminam em prática interprofissional colaborativa, aspectos estes que fornecem o suporte para minimizar as fragilidades dos processos formativos e viabilizar o efetivo trabalho em equipe e a colaboração interprofissionalidade.

Na análise temática do Eixo Temático 2 - Formação em Saúde para a prática Interprofissional, foram encontradas 65 Unidades de Contexto (UC), e 74 Unidades de Registro (UR). A partir das UR emergiram três categorias: Integração ensino-serviço-comunidade; Integralidade no cuidado; e, Currículo, inovações e formação em saúde.

Na categoria Integração ensino-serviço-comunidade (20 UR) emergiram os seguintes conteúdos:

[...] Atualmente, as práticas interprofissionais acontecem muito em projetos de extensão onde unimos extensão, ensino e pesquisa. (P1)

[...] As iniciativas ligadas à extensão, estágios e processos de Residências multiprofissionais representam espaços disparadores para a prática interprofissional no âmbito da formação docente. (P66)

[...] Utilizar espaços de discussões para favorecer o estabelecimento de metas e objetivos que perpassam todo o serviço, incluindo a participação de profissionais dos serviços, docentes, discentes, usuários e familiares.

Os conteúdos retratam a importância da integração ensino-serviço-comunidade para a interprofissionalidade e a integralidade do cuidado.

A integração ensino-serviço promove a troca de saberes e favorece a busca de atualização profissional. Enquanto o docente contribui na identificação de problemas da assistência e propõe estratégias de superação, o profissional da assistência exerce importante papel no fornecimento de informações sobre a realidade em que atua. $\frac{33}{3}$

Para o campo da EIP, em que os profissionais devem aprender uns com os outros e entre si, torna-se essencial que estes profissionais possam desenvolver competências específicas para o trabalho interprofissional. A graduação em saúde e a educação permanente são espaços promissores e essenciais para o preparo de profissionais, capazes de desenvolver 
uma prática interprofissional com vistas à integralidade do cuidado, como também, promover a integração ensino, serviços e comunidade, que são imprescindíveis para a constituição de redes de atenção, redes de saberes e redes de fazeres. Constata-se, assim, a necessidade urgente em reorganizar o trabalho em saúde e reordenar a formação de recursos humanos.

A reorganização precisa unir de forma dialógica e horizontal, o campo da formação profissional e do trabalho. O processo é coletivo e está em construção, nele cada ator tem um importante papel e distintas responsabilidades. .44

Na perspectiva da EIP, o desenvolvimento de competências (específicas ou complementares, comuns e colaborativas) requer a intencionalidade explicitada nos processos formativos.

Conforme descrito por Barr ${ }^{26}$ e Batista ${ }^{35}$, as competências específicas são aquelas que asseguram as identidades profissionais das profissões, subsidiadas pelos marcos teóricos-conceituais e metodológicos que fundamentam as práticas profissionais. As competências comuns são aquelas que marcam a interseção entre todas as profissões. São competências em que as diferentes categorias profissionais podem desenvolver sem interferências nos próprios limites profissionais e dos demais. As competências colaborativas são aquelas que melhoram as relações entre as diferentes categorias profissionais na dinâmica do trabalho em saúde

Desse modo, é importante notar que para o efetivo trabalho em saúde, no âmbito da interprofissionalidade, é necessário que o profissional do futuro seja formado com base nos princípios da EIP. As instituições de ensino superior e os serviços, também, possuem responsabilidade mútuas com esta formação, qualificação e espaços para construção coletiva entre unidades formadoras, serviços, gestão e usuário. Destacase aqui, a escassez de pesquisas envolvendo usuários e familiares como centro deste processo de cuidado.

Na categoria Integralidade no cuidado (40 UR) emergiram os seguintes conteúdos:

[...] Hoje não consigo me enxergar sem trabalhar de forma interprofissional, acredito que somente dessa forma se pode atingir a integralidade do cuidado em saúde. (P29)
[...] Trabalho sempre em equipe, pautado no cuidado integral do paciente, compreendendo o ambiente em que o mesmo está inserido, sendo coparticipante no processo de prevenção e reabilitação. (P59)

[...] Incluir o paciente, garantir visibilidade a este ator, personagem, sujeito, usuário, o cidadão como indivíduo e não apenas como condição clínica que interessa para

o aprendizado, mas como pessoa que tem interesses também, concretos e pragmáticos e para além disso, subjetivos. Enfim, acolhê-lo, ouvi-lo e respeitá-lo, cuidá-

Io, portanto. (P94)

Os relatos retratam que o trabalho em equipe, a parceria entre profissionais e familiares a integração entre os profissionais são essenciais para atingir a integralidade no cuidado em saúde, pois entende-se que um profissional sozinho, na sua área específica de conhecimentos, não conseguirá atingir o olhar integral e ampliado sobre as demandas da pessoa sob cuidado.

A integralidade é um dos princípios doutrinários do SUS e compreende o fazer saúde como algo comple$\mathrm{xo}$, onde interagem aspectos ambientais, emocionais, orgânicos e sociais. O conceito de saúde é ampliado, indo além da doença, considerando os determinantes e condicionantes sociais da saúde dos usuários. Assim, torna um desafio trabalhar a interprofissionalidade e a intersetorialidade. $\underline{\underline{36}}$

O SUS contempla em sua base constitucional importantes elementos que favorecem a implementação da educação e prática interprofissional, visto que nele estão presentes os princípios universalidade do acesso, integralidade, participação social e o trabalho baseado em equipe. ${ }^{37} \mathrm{~A}$ educação e prática interprofissional assumem singular importância neste contexto, compreendendo que o SUS é interprofissional ${ }^{38}$, considerando seus princípios, sobretudo com o advento da Atenção Primária à Saúde que, por meio da ESF, enquanto modelo de reorientação, incorpora diferentes profissões em equipes para atuação compartilhada. $\underline{39}$

Desse modo, percebe-se que os participantes compreendem a importância da EIP e das práticas colaborativas para a integralidade da atenção e que o paciente/usuário deve ser o ator principal neste contexto de saúde, considerando o conceito ampliado de saúde do SUS. 
$\mathrm{Na}$ categoria Currículo, inovações e formação em saúde (14 UR) emergiram os seguintes conteúdos:

\section{[...] Uma sugestão drástica seria uma base curricular comum para todos os cursos de saúde que se desdobrasse para as especialidades das formações à medida que o aluno avançasse na sua formação. (P3)}

\section{[...] Precisamos promover Educaçäo Interprofissional, onde os alunos de diferentes cursos possam se encontrar e trabalhar juntos, ainda na graduação.}

\section{[...] É preciso haver uma mudança do paradigma tradicional de ensino, com vistas à formação de profissionais de saúde que tenham consciência de serem integrantes de uma rede de cuidados $e$ que tenham habilidades suficientes para elaborar planos de cuidado na perspectiva da integralidade, intersetorialidade e no trabalho em equipe. (P65)}

Os conteúdos expressos apontam para a necessidade de mudanças curriculares nas graduações dos cursos da saúde, no intuito de favorecer profissionais com uma formação voltada para a interprofissionalidade e com capacidade para atuar em equipes colaborativas. Os relatos dos participantes indicam a necessidade de aprimorar a formação tanto na graduação como na Educação Permanente, reconhecem fragilidades na formação e mostram-se disponíveis para implementar mudanças.

De acordo com Batista 25 "a vivência de aprendizagens interativas na EIP é reconhecida como promotora do desenvolvimento de competências para a prática colaborativa". Estas experiências precisam ser tomadas como curriculares, evitando classificá-las como optativas e, assim, vistas como de menor importância na formação do futuro profissional de saúde.

Neste contexto, o docente tem um lugar de destaque como mediador nas situações de aprendizagem no contexto da EIP. Desse modo, o docente também precisa estar preparado e ter a intencionalidade para promover reflexões e implementar mudanças curriculares, curricularização das ações de extensão e junto aos estudantes, sendo o facilitador das aprendizagens compartilhadas e das vivências interprofissionais nos cenários de prática.

Acredita-se que com a proposta do currículo integrado, interdisciplinar e interprofissional, os estudantes possam vivenciar e perceber a importância da prática colaborativa ainda na graduação, na perspectiva de tornarem-se futuros profissionais aptos para o trabaIho em equipe e a integralidade da atenção.

\section{Considerações finais}

Observa-se que os dois eixos temáticos "Importância, desafios e possibilidades da Educação e Trabalho Interprofissional" e "Formação em Saúde para a prática Interprofissional" trataram da importância da EIP tanto na prática profissional quanto na formação em saúde. Percebe-se que quando existe um trabalho e/ ou um estudo compartilhado, as ações e/ou aprendizados intervém uns com os outros, proporcionando os modos pelos quais uns colaboram com os outros e tendo como fruto a integralidade da atenção e a interprofissionalidade nas ações.

Este estudo analisou as potencialidades e fragilidades da educação e do trabalho interprofissional na perspectiva de profissionais da saúde do Nordeste brasileiro. Os profissionais apontam a necessidade de aprimoramento e/ou desenvolvimento de diferentes competências colaborativas e relatam dificuldades enfrentadas no cuidado integral ao paciente/ usuário, as barreiras para realizar o diálogo em equipe e a ajuda mútua para a prática colaborativa interprofissional. Além disso, identificam os déficits na formação acadêmica e educação permanente para o fortalecimento da EIP e, consequentemente, para o fortalecimento das práticas interprofissionais nos cenários do trabalho em saúde.

Um desafio envolve a realização de estudos longitudinais de melhorias do trabalho em equipe e da colaboração interprofissional, o que poderá fornecer informações valorosas sobre o modo como uma equipe trabalha e atua nos contextos de atenção à saúde e subsidiar o desenvolvimento de ações de educação permanente para o fortalecimento da força de trabaIho em saúde para maior resolutividade das demandas existentes nas realidades brasileiras.

Outros desafios dizem respeito ao planejamento, implementação e disseminação de processos avaliativos que permitam acompanhar os resultados das iniciativas de EIP tomando como participantes, além de docentes, estudantes e profissionais de saúde, os usuários, familiares e comunidades. $\mathrm{O}$ envolvimento ativo nos processos avaliativos aprimora a conscientização 
sobre a EIP e informa o desenvolvimento das ações, nas quais todos devem estar diretamente envolvidos, com a expectativa de ampliar o entendimento do que a EIP pode, faz e deverá fazer melhor no futuro.

\section{Contribuições das autoras}

Bispo EPF participou da coleta de dados da pesquisa, da concepção, delineamento, busca e análise dos dados da pesquisa, interpretação dos resultados, redação do artigo científico. Rossit RAS participou da interpretação dos dados, concepção, delineamento, análise dos dados da pesquisa, interpretação dos resultados e redação do artigo científico.

\section{Conflitos de interesses}

Nenhum conflito financeiro, legal ou político envolvendo terceiros (governo, empresas e fundações privadas, etc.) foi declarado para nenhum aspecto do trabalho submetido (incluindo, mas não se limitando a subvenções e financiamentos, participação em conselho consultivo, desenho de estudo, preparação de manuscrito, análise estatística, etc.).

\section{Referências}

1. Reeves S. Porque precisamos da educação interprofissional para um cuidado efetivo e seguro. Interface (Botucatu). 2016;20(56):185-96. https://doi.org/10.1590/1807-57622014.0092

2. Organização Mundial de Saúde. Marco para Ação em Educação Interprofissional e Prática Colaborativa [Internet]. Genebra: OMS; 2010. Disponível em: http://www.fnepas.org.br/oms traduzido_2010.pdf

3. Barr H. Competent to collaborate; towards a competency-based model for Interprofessional education. J. Interprof. Care [Internet]. 1998;1(2):181-8. Disponível em: https://neipc.ufes.br/sites/neipc. ufes.br/files/field/anexo/competent to collaborate.pdf

4. Rossit RAS, Freitas MAO, Batista SSHS, Batista NA. Construção da identidade profissional na Educação Interprofissional em Saúde: percepção de egressos. Interface (Botucatu). 2018;22(1):1399-410. https://doi.org/10.1590/1807-57622017.0184

5. Reeves S, Perrier L, Goldman J, Freeth D, Zwarenstein M. Interprofessional education: effects on professional practice and healthcare outcomes (update). Cochrane Database Syst Rev. 2013;2013(3):CD002213. https://doi.org/10.1002/14651858. cd002213.pub3
6. Reeves S, Xyrichis A, Zwarenstein M. Teamwork, collaboration, coordination, and networking: Why we need to distinguish between different types of interprofessional practice. J Interprof Care. 2018;32(1):1-3. https://doi.org/10.1080/13561820.2017.140 $\underline{0150}$

7. Peduzzi M, Agreli HLF, Silva JAM, Souza HS. Trabalho em equipe: uma revisita ao conceito e a seus desdobramentos no trabalho interprofissional. Trab. Educ. Saúde. 2020;18(suppl 1):e0024678. https://doi.org/10.1590/1981-7746-sol00246

8. Willgerodt MA, Abu-Rish Blakeney E, Brock DM, Liner D, Murphy $\mathrm{N}$, Zierler B. Interprofessional education and practice guide No. 4: Developing and sustaining interprofessional education at an academic health center. J Interprof Care. 2015;29(5):421-5. https:// doi.org/10.3109/13561820.2015.1039117

9. Peduzzi M, Norman I, Coster S, Meireles E. Adaptação transcultural e validação da Readiness for Interprofessional Learning Scale no Brasil. Rev. Esc. Enferm. USP. 2015;49:7-15. https://doi.org/10.1590/S0080-623420150000800002

10. Ministério da Saúde. Humaniza SUS - Política Nacional de Humanização [Internet]. Brasília: Ministério da Saúde; 2003. Disponível em: https://bvsms.saude.gov.br/bvs/publicacoes/ politica_nacional_humanizacao_pnh_folheto.pdf

11. Minayo MCS. Amostragem e saturação em pesquisa qualitativa: consensos e controvérsias. Revista Pesquisa Qualitativa [Internet]. 2017;5(7):01-12. Disponível em: https:// editora.sepq.org.br/index.php/rpq/article/download/82/59

12. Bispo EPF, Rossit RAS. Adaptação transcultural e validação estatística do Assessment of Interprofessional Team Collaboration Scale II. Revista Ágape [Internet]. 2018;1(1):1-15. Disponível em: http://revistaagape.com.br/index.php/revistaagape/article/ download/22/3

13. Minayo MC, organizadora. Pesquisa social: Teoria, método e criatividade. $29^{\mathrm{a}}$ ed. Petrópolis: Vozes.

14. Franco MLPB. Análise de Conteúdo. $2^{\mathrm{a}}$ ed. Brasília: Liber Livro; 2005.

15. Minayo MCS, Gomes SFD. Pesquisa Social: Teoria, método e criatividade. 30ª ed. Petrópolis: Vozes; 2011.

16. Health Professions Accreditors Collaborative. Guidance on developing quality interprofessional education for the health professions [Internet]. Chicago, IL: Health Professions Accreditors Collaborative; 2019. Disponível em: https:// healthprofessionsaccreditors.org/wp-content/uploads/2019/02/ HPACGuidance02-01-19.pdf

17. Canadian Interprofessional Health Collaborative. A National Interprofessional Competency Framework [Internet]. Vancouver: CIHC; 2010.Disponível em: http://ipcontherun.ca/wp-content/ uploads/2014/06/National-Framework.pdf 
18. Interprofessional Education Collaborative Expert Panel. Core competencies for interprofessional practice: report on an expert panel [Internet]. Washington: Interprofessional Education Collaborative; 2011. Disponível em: https://www.aacom.org/docs/ default-source/insideome/ccrpt05-10-11.pdf?sfvrsn=77937f97_2

19. Rocha FAA, Barreto ICHC, Moreira AE.M.M. Colaboração interprofissional: estudo de caso entre gestores, docentes e profissionais de saúde da família. Interface (Botucatu). 2016;20(57):415-26. https://doi.org/10.1590/1807-57622015.0370

20. Dias MS, Vieira FM, Silva LC, Vasconcelos MI, Machado MD. Colaboração interprofissional no projeto saúde e prevenção na escola. Ciênc saúde colet. 2016;21(6):1789-98. https://doi. org/10.1590/1413-81232015216.08112016

21. Peduzzi M, Norman IJ, Germani AC, Silva JA, Souza GC. Educação interprofissional: formação de profissionais de saúde para o trabalho em equipe com foco nos usuários. Rev Esc Enferm USP. 2013;47(4):977-83. https://doi.org/10.1590/S0080623420130000400029

22. Costa, MV. A educação interprofissional no contexto brasileiro: algumas reflexões. Interface Comunicação Saúde Educação. 2016;20(56):197-8. https://doi.org/10.1590/1807-57622015.0311

23. Silva JA, Peduzzi M, Orchard C, Leonello VM. Educação interprofissional e prática colaborativa na Atenção Primária à Saúde. Rev Esc Enferm USP. 2015;49(2):16-24. https://doi. org/10.1590/S0080-623420150000800003

24. Shrader S, Hodgkins R, Laverentz D, Zaudke J, Waxman $\mathrm{M}$, Johnston $\mathrm{K}$, et al. Interprofessional Education and Practice Guide No. 7: Development, implementation, and evaluation of a large-scale required interprofessional education foundational programme. Journal of interprofessional care. 2016;30(5):615-9. https://doi.org/10.1080/13561820.2016.1189889

25. Batista NA, Batista SH. Educação interprofissional na formação em saúde: tecendo redes de práticas e saberes. Interface (Botucatu) [Internet]. 2016;20:202-4. Disponível em: https://www. scielo.br/j/icse/a/kh54yfRbjcFfdzmGXj8rP3M/?lang=pt\&format=pdf

26. Barr $\mathrm{H}$, Low $\mathrm{H}$. Interprofessional learning in pre-registration education courses: A CAIPE guide for commissioners and regulators of education. London, CAIPE: 2013.

27. Araújo TA, Vasconcelos AC, Pessoa TR, Forte FD.

Multiprofissionalidade e interprofissionalidade em uma residência hospitalar: o olhar de residentes e preceptores. Interface (Botucatu). 2017;21(62):601-13. https://doi.org/10.1590/1807$\underline{57622016.0295}$

28. Oandasan I, Reeves S. Key elements for interprofessional education. Part 1: the learner, the educator and the learning context. J Interprof Care. 2005;19(suppl 1):21-38. https://doi. org/10.1080/13561820500083550
29. Costa MVA. Apotência da educação interprofissional para o desenvolvimento de competências colaborativas no trabalho em saúde. In: Toassi RFC, organizadora. Interprofissionalidade e formação na saúde: onde estamos?. Porto Alegre: Rede UNIDA; 2017.

30. Peduzzi M. Educação Interprofissional para o desenvolvimento de competências colaborativas em saúde. In: Toassi RFC, organizadora. Interprofissionalidade e formação na saúde: onde estamos?. Porto Alegre: Rede Unida; 2017.

31. Stone N. Coming in from the interprofessional cold in Australia. Aust Health Rev. 2007;31(3):332-40. https://doi. org/10.1071/ah070332

32. Freeth DS, Hammick M, Reeves S, Koppel I, Barr H. Effective interprofessional education: development, delivery and evaluation [Internet]. Oxford: Blackwell with CAIPE; 2005. Disponível em: https://onlinelibrary.wiley.com/doi/book/10.1002/9780470776438

33. Beccaria LM, Trevizan MA. Ações do processo de integração docente assistencial entre um curso de graduação em enfermagem e um hospital de ensino. Arq. cienc saúde [Internet]. 2006;13(2):89-96. Disponível em: https://repositorio-racs.famerp. br/racs ol/vol-13-2/06-ID\%20185.pdf

34. Brehmer LCF, Ramos FRS. Experiências de integração ensinoserviço no processo de formação profissional em saúde: revisão integrativa. Rev. Eletr. Enf. 2014;16(1):228-37. http://dx.doi. org/10.5216/ree.v16i1.20132

35. Batista NA. Educação Interprofissional em Saúde: Concepções e Práticas. Cad Fnepas [Internet]. 2012;2(1):25-8. Disponível em: http://fnepas.org.br/artigos caderno/v2/educacao interprofissional.pdf

36. Ellery AEL. Interprofissionalidade na Estratégia Saúde da Família: condições de possibilidade para a integração de saberes e a colaboração interprofissional. Interface. 2014;18(48):213-5. https://doi.org/10.1590/1807-57622013.0387

37. Portaria $n^{\circ}$. 2488, de 21 de outubro de 2011 (Brasil). Aprova a Política Nacional de Atenção Básica, estabelecendo a revisão de diretrizes e normas para a organização da Atenção Básica, para a Estratégia Saúde da Família (ESF) e o Programa de Agentes Comunitários de Saúde (PACS) [Internet]. Diário Oficial da União. https://bvsms.saude.gov.br/bvs/saudelegis/gm/2011/ prt2488_21_10_2011.html

38. Peduzzi M. O SUS é interprofissional. Interface. 2016;20(56):199-201. https://doi.org/10.1590/1807-57622015.0383

39. Barr H. Interprofessional education: the genesis of a global movement. Londres: Centre for Advancement of Interprofessional Education; 2015. 